November 6, 2002

\title{
A Simple Axiomatization and Constructive Representation Proof for Choquet Expected Utility*
}

\begin{abstract}
We provide a set of simple and intuitive set of axioms that allow for a direct and constructive proof of the Choquet Expected Utility representation for decision making under uncertainty.
\end{abstract}

\author{
Alain Chateauneuf \\ CERMSEM \\ Université Paris I \\ Jürgen Eichberger \\ Wirtschaftstheorie I \\ Universität Heidelberg \\ Simon Grant \\ Department of Economics \\ Rice University
}

${ }^{*}$ We thank Matthew Ryan for very useful comments and suggestions on related work and for encouraging us to write this note. 


\section{Introduction}

In subjective expected utility theory choice under uncertainty is perceived as the maximization of the mathematical expectation of a utility function with respect to the subjective probabilities that represent the individual's subjective assessment of the relative likelihood of events (see Savage [1954] for the definitive statement and Anscombe and Aumann [1963] for an alternative, simpler, treatment.) Motivated by examples such as the well-known Ellsberg paradoxes and an increasing body of experimental and empirical evidence, decision theorists have developed and analyzed models for decision making under uncertainty that entail the mathematical expectation of a utility function with respect to "non-additive probabilities' or capacities. This generalization of subjective expected utility has been dubbed Choquet Expected Utility as it utilizes the Choquet integral for non-additive measures (see Gilboa [1989] for an axiomatization in the Savage purely subjective uncertainty setting and Schmeidler [1989] for an alternative and simpler treatment in the Anscombe and Aumann setting.)

In this note we adopt the Anscombe and Aumann setting of the horse/roulette lotteries representation of alternative courses of action. That is, courses of action, or acts, are functions from a set of states of nature to the set of simple lotteries defined over an arbitrary set of outcomes. Our aim is to exploit this rich structure to provide a set of simple and intuitive axioms that allow us to provide a direct and constructive proof of the Choquet Expected Utility representation. Nothing that we do constitutes a technical advance on Schmeidler's (1989) pioneering work but we submit that our constructive proof allows a more direct and intuitive comparison with the standard Expected Utility model. Indeed to quote Anscombe and Aumann (1963)

"The novelty of our presentation, if any, lies in the double use of [expected] utility theory, permitting the very simple and plausible assumptions and the simple construction and proof." (p. 203)

\section{The Set-up and the Axioms}

We set our analysis in the context of the well-known Anscombe-Aumann framework (Anscombe \& Aumann (1962)). We assume that the uncertainty a decision maker faces can be described by a non-empty set of states, denoted by $\mathcal{S}$. This set may be finite or infinite. Associated with the set of states is the set of events, taken to be a sigma-algebra of subsets of $\mathcal{S}$, denoted by $\mathcal{E}$. We assume that for each $s$ in $\mathcal{S},\{s\}$ is in $\mathcal{E}$. Let $\mathcal{X}$ be an arbitrary non-empty set of outcomes (finite or infinite) and denote by $\mathcal{L}$ the set of lotteries (simple probability measures) on $\mathcal{X}$. An act, or horse-race lottery, is a function (measurable with respect to $\mathcal{E}$ ) 
$f: \mathcal{S} \rightarrow \mathcal{L}$ with finite range, $\mathcal{F}$ denotes the set of such acts. We shall identify each $L \in \mathcal{L}$ with the constant horse-race lottery $[L$ on $\mathcal{S}]$ (that is, $f(s)=L$ for all $s \in \mathcal{S}$ ) and so with slight abuse of notation, we shall let $\mathcal{L}$ also denote the set of constant acts. For each $x$ in $\mathcal{X}$, let $\delta_{x}$ denote the (degenerate) lottery that yields the outcome $x$ with probability one.

Let $\succsim$ denote the individual's preference relation on $\mathcal{F}$. For ease of exposition and clarity we impose without essential loss of generality a structural assumption that there exists a maximally preferred outcome and a minimally preferred outcome.

Axiom 0 Their exist two outcomes $M$ and 0 , such that $\delta_{M} \succ \delta_{0}$, and $\delta_{M} \succsim \delta_{x} \succsim \delta_{0}$, for all $x \in \mathcal{X}$

Both the sets $\mathcal{L}$ and $\mathcal{F}$ are mixture spaces. In particular, for any pair of lotteries $L^{\prime}$ and $L^{\prime \prime}$ in $\mathcal{L}$, and any $\alpha$ in $(0,1), \alpha L^{\prime}+(1-\alpha) L^{\prime \prime}$ is the lottery $L$ in $\mathcal{L}$ for which $L(x)=$ $\alpha L^{\prime}(x)+(1-\alpha) L^{\prime \prime}(x)$, for each $x$ in $\mathcal{X}$. Similarly, for any pair of horse-race lotteries $f^{\prime}$ and $f^{\prime \prime}$ in $\mathcal{F}$, and any $\alpha$ in $(0,1)$, we define $\alpha f^{\prime}+(1-\alpha) f^{\prime \prime}$ to be the horse-race lottery $f \in \mathcal{F}$, for which $f(s)=\alpha f^{\prime}(s)+(1-\alpha) f^{\prime \prime}(s)$, for each $s$ in $\mathcal{S}$. A utility function $U: \mathcal{L} \rightarrow \mathbb{R}$ defined on the lottery space is said to be affine if for any pair of lotteries $L^{\prime}$ and $L^{\prime \prime}$ in $\mathcal{L}$, and any $\alpha$ in $(0,1), U\left(\alpha L^{\prime}+(1-\alpha) L^{\prime \prime}\right)=\alpha U\left(L^{\prime}\right)+(1-\alpha) U\left(L^{\prime \prime}\right)$.

Capacities are real-valued functions defined on $\mathcal{E}$, that generalize the notion of a probability measure. Formally, a capacity is a normalized set function.

Definition 1. A capacity is a function $\nu: \mathcal{E} \rightarrow \mathbb{R}$ that assigns real numbers to events, such that

(i) $E, F \in \mathcal{E}, E \subseteq F$ implies $\nu(E) \leq \nu(F)$, (monotonicity)

(ii) $\nu(\varnothing)=0$ and $\nu(\mathcal{S})=1$ (normalization).

A probability measure $\mu: \mathcal{E} \rightarrow \mathbb{R}$ is a capacity that also satisfies the following property

(iv) $E, F \in \mathcal{E}, \mu(E \cup F)=\mu(E)+\mu(F)-\mu(E \cap F)$ (additivity).

In order to define the Choquet integral with respect to a capacity and to compare it with the usual Lebesgue integral taken with respect to a probability measure, it is convenient to associate with an act $f \in \mathcal{F}$, the coarsest (finite) partition over $\mathcal{S}$, of the form $\left\{E_{1}, \ldots, E_{n}\right\}$ to which the act $f$ is measurable and ordered. That is, for any pair of states $s, t \in S$, if both $s$ and $t$ are in some $E \in\left\{E_{1}, \ldots, E_{n}\right\}$ then $f(s)=f(t)$, otherwise $f(s) \neq f(t)$. Furthermore for any $s \in E_{i}$ and $t \in E_{j}, i<j$ implies $f(s) \succsim f(t)$. For each $i=1, \ldots, n$, if we let $L_{i}$ be the lottery prize resulting if a state in $E_{i}$ obtains, then $f$ may be expressed in the form $f=\left[L_{1}\right.$ on $E_{1} ; \ldots ; L_{n}$ on $\left.E_{n}\right]$. Throughout the rest of this paper, if an act is expressed in this form, then the associated partition should be taken to be one with respect to which that act is measurable and ordered. 
Definition 2. Fix an affine utility function $U: \mathcal{L} \rightarrow \mathbb{R}$, capacity $\nu: \mathcal{E} \rightarrow[0,1]$, and any act $f=\left[L_{1}\right.$ on $E_{1} ; \ldots ; L_{n}$ on $\left.E_{n}\right]$. The Choquet expected utility of the act $f$ with respect to $U$ and $\nu$ is defined to be

$$
\begin{aligned}
\operatorname{CEU}(f) & =\int U \circ f d \nu \\
& =\sum_{i=1}^{n-1}\left(U\left(L_{i}\right)-U\left(L_{i+1}\right)\right) \nu\left(\cup_{j=1}^{i} E_{j}\right)+U\left(L_{n}\right) \\
& =U\left(L_{1}\right) \nu\left(E_{1}\right)+\sum_{i=2}^{n}\left(U\left(L_{i}\right)\right)\left(\nu\left(\cup_{j=1}^{i} E_{j}\right)-\nu\left(\cup_{j=1}^{i-1} E_{j}\right)\right)
\end{aligned}
$$

The expression on the right-hand-side of (2) may be interpreted as saying that the decision weight placed on the event yielding a lottery prize equal in preference to a particular lottery $L$, is equal to the difference between the capacity of the event that yields a lottery prize equal to or better than $L$ and the capacity of the event yielding a strictly better lottery prize. If the capacity in definition 2 is actually a probability measure $\mu($.$) (that is, it also$ satisfies additivity) then the expression on the right-hand-side of (2) collapses to the standard expected utility formulation, $\operatorname{SEU}(f)=\sum_{i=1}^{n} U\left(L_{i}\right) \mu\left(E_{i}\right)$.

Notice that, when restricted to the set of constant acts, the Choquet Expected Utility functional is simply $U($.$) and so is affine. If we normalize this utility function by setting$ $U\left(\delta_{M}\right):=1$ and $U\left(\delta_{0}\right):=0$, then the CEU representation may be viewed as involving the assignment to each lottery $L$ a utility equal to the probability $U(L)$ which makes the lottery $U(L) \delta_{M}+(1-U(L)) \delta_{0}$ indifferent to $L$. Furthermore, the CEU representation also entails the assignment to each event $E \in \mathcal{E}$, a capacity equal to the probability $\nu(E)$ which makes the constant act $\nu(E) \delta_{M}+(1-\nu(E)) \delta_{0}$ indifferent to the act $\left[\delta_{M}\right.$ on $E ; \delta_{0}$ on $\left.E\right]$.

For a simple example illustrating how these assignments can be combined to construct the Choquet Expected Utility of an act, consider the act $g=\left[L\right.$ on $A ; L^{\prime}$ on $\left.\mathcal{S}-A\right]$. Suppose $L \sim U(L) \delta_{M}+(1-U(L)) \delta_{0}, L^{\prime} \sim U\left(L^{\prime}\right) \delta_{M}+\left(1-U\left(L^{\prime}\right)\right) \delta_{0}$. Since by our convention $L \succsim L^{\prime}$, we have $U(L) \geq U\left(L^{\prime}\right)$. So, if we replace $L$ by $U(L) \delta_{M}+(1-U(L)) \delta_{0}$ and $L^{\prime}$ by $U\left(L^{\prime}\right) \delta_{M}+\left(1-U\left(L^{\prime}\right)\right) \delta_{0}$ in $g$, we form the act $g^{\prime}=$

$$
\begin{gathered}
{\left[\begin{array}{cc}
U(L) \delta_{M}+(1-U(L)) \delta_{0} & \text { on } A \\
U\left(L^{\prime}\right) \delta_{M}+\left(1-U\left(L^{\prime}\right)\right) \delta_{0} & \text { on } \mathcal{S}-A
\end{array}\right]=} \\
{[1-U(L)]\left[\begin{array}{cc}
\delta_{0} & \text { on } A \\
\delta_{0} & \text { on } \mathcal{S}-A
\end{array}\right]+\left[U(L)-U\left(L^{\prime}\right)\right]\left[\begin{array}{cc}
\delta_{M} & \text { on } A \\
\delta_{0} & \text { on } \mathcal{S}-A
\end{array}\right]+U\left(L^{\prime}\right)\left[\begin{array}{cc}
\delta_{M} & \text { on } A \\
\delta_{M} & \text { on } \mathcal{S}-A
\end{array}\right]}
\end{gathered}
$$


Now, suppose further that $\nu(A) \delta_{M}+(1-\nu(A)) \delta_{0} \sim\left[\delta_{M}\right.$ on $A ; \delta_{0}$ on $\left.\mathcal{S}-A\right]$. If we replace $\left[\delta_{M}\right.$ on $A ; \delta_{0}$ on $\left.\mathcal{S}-A\right]$ in $(3)$ by $\nu(A) \delta_{M}+(1-\nu(A)) \delta_{0}$ then the act $g^{\prime}$ is transformed into the constant act

$$
\begin{aligned}
g^{\prime \prime} & =[1-U(L)] \delta_{0}+\left[U(L)-U\left(L^{\prime}\right)\right]\left[\nu(A) \delta_{M}+(1-\nu(A)) \delta_{0}\right]+U\left(L^{\prime}\right) \delta_{M} \\
& =\left(\left[U(L)-U\left(L^{\prime}\right)\right] \nu(A)+U\left(L^{\prime}\right)\right) \delta_{M}+\left(1-\left[U(L)-U\left(L^{\prime}\right)\right] \nu(A)-U\left(L^{\prime}\right)\right) \delta_{0}
\end{aligned}
$$

Notice that the probability weight on $\delta_{M}$ for $g^{\prime \prime}$ is the Choquet expected utility of $g$ according to the rule given by the expression in the right-hand-side of (1). The following axioms provide a set of conditions that are both necessary and sufficient to allow us to infer that $g \sim g^{\prime}$ and $g^{\prime} \sim g^{\prime \prime}$ and so (by transitivity of indifference) that $g \sim g^{\prime \prime}$, thereby allowing us to make the assignment $\mathrm{CEU}(g):=\left[U(L)-U\left(L^{\prime}\right)\right] \nu(A)+U\left(L^{\prime}\right)$.

We begin with the standard ordering axiom and the most elementary forms of continuity and monotonicity axioms for the preference relation.

\section{Axiom 1 (Ordering)}

The preference relation $\succsim$ is complete, reflexive and transitive.

For any pair of outcomes $x$ and $y$ in $\mathcal{X}$, let $\mathcal{L}_{\{x, y\}}$ denote the set of lotteries whose support is a subset of $\{x, y\}$. We only require the following weak Archimedean property with respect to probability mixtures of two lotteries which share a common two-outcome support.

\section{Axiom 2 ([Two-outcome] Mixture Continuity).}

For any pair of outcomes $x, y \in \mathcal{X}$ and any pair of roulette lotteries $L^{\prime}, L^{\prime \prime} \in \mathcal{L}_{\{x, y\}}$, and any act $f \in \mathcal{L}$, where $L^{\prime} \succ f \succ L^{\prime \prime}$, there is $\alpha, \beta \in(0,1)$ such that $\alpha L^{\prime}+(1-\alpha) L^{\prime \prime} \succ f \succ$ $\beta L^{\prime}+(1-\beta) L^{\prime \prime}$.

We impose the simplest monotonicity axiom for lotteries: if two lotteries have a common two-outcome support, then the one that places more weight on the preferred outcome ranks higher.

\section{Axiom 3 ([Two-outcome] Mixture Monotonicity).}

For any pair of outcomes $x, y \in \mathcal{X}$, such that $\delta_{x} \succ \delta_{y}$, any pair of numbers $\alpha, \beta \in(0,1]$, $\alpha \delta_{x}+(1-\alpha) \delta_{y} \succcurlyeq \beta \delta_{x}+(1-\beta) \delta_{y} \Leftrightarrow \alpha \geq \beta$.

We also impose the simplest notion of set dominance for elementary bets: if $A$ is a subset of $B$ then 'betting on $A$ ' is not strictly preferred to 'betting on $B$ '.

Axiom 4 (Event Dominance).

For any pair of outcomes $x, y \in \mathcal{X}$, such that $\delta_{x} \succ \delta_{y}$, and any pair of events $A, B \in \mathcal{E}$, such that $A \subset B,\left[\delta_{x}\right.$ on $B ; \delta_{y}$ on $\left.\mathcal{S}-B\right] \succcurlyeq\left[\delta_{x}\right.$ on $A ; \delta_{y}$ on $\left.\mathcal{S}-A\right]$. 
The last two axioms are the principle substitution axioms, that in conjunction with the first four, permit us to apply von Neumann-Morgenstern's theorem twice. The first application enables us to calibrate the utility of lotteries and the second enables us to calibrate the capacity of every event, in the manner suggested above. More specifically, the axiom, Lottery Substitution of Indifference, considers the replacement of a lottery prize on an event in an act with an equally desirable lottery prize. Such a replacement is assumed to leave the overall desirability of the act unaffected.

Axiom 5 (Lottery Substitution of Indifference).

For any act $f=\left[L_{1}\right.$ on $E_{1} ; \ldots ; L_{n}$ on $\left.E_{n}\right] \in \mathcal{F}$, and lottery $L^{\prime}$ and any $i=1, \ldots, n$, if $L^{\prime} \sim L_{i}$ then $f \sim\left[L_{1}\right.$ on $E_{1} ; \ldots ; L_{i-1}$ on $E_{i-1} ; L^{\prime}$ on $E_{i} ; L_{i+1}$ on $E_{i+1} ; \ldots ; L_{n}$ on $\left.E_{n}\right]$.

The final axiom, is the independence axiom restricted to pairs of comonotonic acts belonging to the same indifference class taking mixtures of a third act that is pairwise comonotonic with the other two.

Axiom 6 (Comonotonic Independence of Indifference)

For any $\alpha$ in $(0,1]$, and any three acts $f, g, h \in \mathcal{F}$ such that $f(s) \succ f(t)$ implies $g(s) \succsim g(t)$, $g(s) \succ g(t)$ implies $h(s) \succsim h(t)$, and $h(s) \succ h(t)$ implies $f(s) \succsim f(t)$, for all $s, t \in S$; if $f \sim g$ then $\alpha f+(1-\alpha) h \sim \alpha g+(1-\alpha) h$.

\section{The Representation and Proof}

Theorem 1 Suppose $\succsim$ satisfies Axiom 0, then the following two statements are equivalent:

(a) The preference relation $\succsim$ on $\mathcal{F}$ satisfies Axioms 1-6, that is, Ordering, Mixture Continuity, Mixture Monotonicity, Event Dominance, Lottery Substitution of Indifference and Comonotonic Independence of Indifference.

(b) There exists a unique capacity $\nu$ on $\mathcal{E}$ and a unique affine real-valued function $U$ on $\mathcal{L}$, with $U\left(\delta_{M}\right)=1$ and $U\left(\delta_{0}\right)=0$, such that for all $f, g \in \mathcal{F}$

$$
f \succsim g \Leftrightarrow \int U \circ f d \nu \geq \int U \circ g d \nu
$$

Proof. We begin with a preliminary result that states that if an act lies in preference between a pair of degenerate lotteries, then there is a unique probability mixture of those two lotteries which is indifferent to that act. 
Lemma 2 ([Two-outcome] Mixture Solvability) Axioms 1-3 (that is, Ordering, Mixture Continuity and Mixture Monotonicity) imply that $\succsim$ satisfies the following solvability property: for any pair of outcomes $x, y \in \mathcal{X}$, such that $\delta_{x} \succ \delta_{y}$, and any act $f \in \mathcal{F}$, such that $\delta_{x} \succsim f \succsim \delta_{y}$, there exists a unique $\alpha$ in $[0,1]$, such that $\alpha \delta_{x}+(1-\alpha) \delta_{y} \sim f$.

For a proof see FishBURN (1979), Lemma 3.1, p.33.

We are now in a position to show (a) implies (b), by explicitly constructing the CEUrepresentation for $\succsim$. We proceed by first deriving an Expected Utility representation for the preference relation restricted to the set of constant acts. That is, we construct the affine realvalued function $U$ defined on $\mathcal{L}$. In the second step, we use this $U$ to calibrate the decision weights on events to construct the capacity $\nu$ defined on $\mathcal{E}$, that enables us to extend the representation to the entire set of acts.

Step 1. Constructing the EU-Representation on $\succsim$ restricted to $\mathcal{L}$, the set of constant acts.

Set $U\left(\delta_{M}\right):=0$ and $U\left(\delta_{0}\right):=1$. For any $y \in \mathcal{X}$ set $U\left(\delta_{y}\right):=\beta$, where, by Lemma 2 (Mixture Solvability), $\beta$ is the unique solution to $\beta \delta_{M}+(1-\beta) \delta_{0} \sim \delta_{y}$. Since all constant acts are pair-wise comonotonic, notice that for any $L=\sum_{i=1}^{m} \alpha_{i} \delta_{x_{i}} \in \mathcal{L}$ we can apply Axiom 6 (Comonotonic Independence of Indifference) and transitivity of indifference (Axiom 1) $m$ times to obtain

$$
\begin{aligned}
L & \sim \alpha_{1}\left(U\left(\delta_{x_{1}}\right) \delta_{M}+\left(1-U\left(\delta_{x_{1}}\right)\right) \delta_{0}\right)+\sum_{i=2}^{m} \alpha_{i} \delta_{x_{i}} \\
& \sim \cdots \sim \sum_{i=1}^{m} \alpha_{i}\left(U\left(\delta_{x_{i}}\right) \delta_{M}+\left(1-U\left(\delta_{x_{i}}\right)\right) \delta_{0}\right) \\
& =\left(\sum_{i=1}^{m} \alpha_{i} U\left(\delta_{x_{i}}\right)\right) \delta_{M}+\left(1-\left(\sum_{i=1}^{m} \alpha_{i} U\left(\delta_{x_{i}}\right)\right)\right) \delta_{0}
\end{aligned}
$$

Hence for any pair of constant acts $L=\sum_{i=1}^{m} \alpha_{i} \delta_{x_{i}}$ and $L^{\prime}=\sum_{j=1}^{m^{\prime}} \beta_{j} \delta_{x_{j}}$, transitivity of preference (Axiom 1) implies $L \succsim L^{\prime}$ if and only if

$$
\begin{aligned}
& \left(\sum_{i=i}^{m} \alpha_{i} U\left(\delta_{x_{i}}\right)\right) \delta_{M}+\left(1-\left(\sum_{i=i}^{m} \alpha_{i} U\left(\delta_{x_{i}}\right)\right)\right) \delta_{0} \\
\succsim & \left(\sum_{j=i}^{m^{\prime}} \beta_{j} U\left(\delta_{x_{j}}\right)\right) \delta_{M}+\left(1-\left(\sum_{j=1}^{m^{\prime}} \beta_{j} U\left(\delta_{x_{j}}\right)\right)\right) \delta_{0} .
\end{aligned}
$$

But by Axiom 3 (Mixture Monotonicity) this holds if and only if

$$
\left(\sum_{i=i}^{m} \alpha_{i} U\left(\delta_{x_{i}}\right)\right) \geq\left(\sum_{j=i}^{m^{\prime}} \beta_{j} U\left(\delta_{x_{j}}\right)\right) .
$$


Hence the affine function $U\left(\sum_{i=1}^{m} \alpha_{i} \delta_{x_{i}}\right)=\sum_{i=1}^{m} \alpha_{i} U\left(\delta_{x_{i}}\right)$ represents $\succsim$ restricted to $\mathcal{L}$.

Step 2. Constructing the CEU-Representation for $\succsim$.

Fix any $f=\left[L_{1}\right.$ on $E_{1} ; \ldots ; L_{n}$ on $\left.E_{n}\right] \in \mathcal{F}$. For each $i=1, \ldots, n$, it follows from Step 1 that there is a unique number $U\left(L_{i}\right) \in[0,1]$, for which

$$
L_{i} \sim U\left(L_{i}\right) \delta_{M}+\left(1-U\left(L_{i}\right)\right) \delta_{x_{*}} .
$$

For each $i=1, \ldots, n-1$, it follows from Lemma 2 that there exists a unique $\nu_{i}$ satisfying

$$
\left[\delta_{M} \text { on } E_{1} \cup \ldots \cup E_{i} ; \delta_{0} \text { on } E_{i+1} \cup \ldots \cup E_{n}\right] \sim \nu_{i} \delta_{M}+\left(1-\nu_{i}\right) \delta_{0} .
$$

From Axiom 3 (Mixture Monotonicity) it follows that $1 \geq U\left(L_{1}\right) \geq \ldots \geq U\left(L_{n}\right) \geq 0$. From Axiom 4 (Event Dominance) it follows that $0 \leq \nu_{1} \leq \ldots \leq \nu_{n-1} \leq 1$. By applying Axiom 5 (Lottery Substitution of Indifference) $n$ times we obtain

$$
\begin{aligned}
& f=\left[\begin{array}{cc}
L_{1} & \text { on } E_{1} \\
\vdots & \vdots \\
L_{n} & \text { on } E_{n}
\end{array}\right] \sim\left[\begin{array}{cc}
U\left(L_{1}\right) \delta_{M}+\left(1-U\left(L_{1}\right)\right) \delta_{0} & \text { on } E_{1} \\
\vdots & \vdots \\
U\left(L_{n}\right) \delta_{M}+\left(1-U\left(L_{n}\right)\right) \delta_{0} & \text { on } E_{n}
\end{array}\right] \\
& =\left(1-U\left(L_{1}\right)\right)\left[\begin{array}{cc}
\delta_{0} & \text { on } E_{1} \\
\delta_{0} & \text { on } E_{2} \\
\delta_{0} & \text { on } E_{3} \\
\vdots & \vdots \\
\delta_{0} & \text { on } E_{n-1} \\
\delta_{0} & \text { on } E_{n}
\end{array}\right]+\left(U\left(L_{1}\right)-U\left(L_{2}\right)\right)\left[\begin{array}{cc}
\delta_{M} & \text { on } E_{1} \\
\delta_{0} & \text { on } E_{2} \\
\delta_{0} & \text { on } E_{3} \\
\vdots & \vdots \\
\delta_{0} & \text { on } E_{n-1} \\
\delta_{0} & \text { on } E_{n}
\end{array}\right]+\cdots \\
& +\left(U\left(L_{n-1}\right)-U\left(L_{n}\right)\right)\left[\begin{array}{cc}
\delta_{M} & \text { on } E_{1} \\
\delta_{M} & \text { on } E_{2} \\
\delta_{M} & \text { on } E_{3} \\
\vdots & \vdots \\
\delta_{M} & \text { on } E_{n-1} \\
\delta_{0} & \text { on } E_{n}
\end{array}\right]+U\left(L_{n}\right)\left[\begin{array}{cc}
\delta_{M} & \text { on } E_{1} \\
\delta_{M} & \text { on } E_{2} \\
\delta_{M} & \text { on } E_{3} \\
\vdots & \vdots \\
\delta_{M} & \text { on } E_{n-1} \\
\delta_{M} & \text { on } E_{n}
\end{array}\right]
\end{aligned}
$$


By applying Axiom 6 (Comonotonic Independence of Indifference) $n-1$ times we have $f$ is indifferent to:

$$
\begin{gathered}
\left(1-U\left(L_{1}\right)\right) \delta_{0}+\left(U\left(L_{1}\right)-U\left(L_{2}\right)\right)\left[\nu_{1} \delta_{M}+\left(1-\nu_{1}\right) \delta_{0}\right]+\left(U\left(L_{2}\right)-U\left(L_{3}\right)\right)\left[\nu_{2} \delta_{M}+\left(1-\nu_{2}\right) \delta_{0}\right] \\
+\ldots+\left(U\left(L_{n-1}\right)-U\left(L_{n}\right)\right)\left[\nu_{n-1} \delta_{M}+\left(1-\nu_{n-1}\right) \delta_{0}\right]+U\left(L_{n}\right) \delta_{M} \\
=\left[\sum_{i=1}^{n-1}\left(U\left(L_{i}\right)-U\left(L_{i+1}\right)\right) \nu_{i}+U\left(L_{n}\right)\right] \delta_{M}+\left[1-U\left(L_{n}\right)-\sum_{i=1}^{n-1}\left(U\left(L_{i}\right)-U\left(L_{i+1}\right)\right) \nu_{i}\right] \delta_{0} .
\end{gathered}
$$

Hence if we take any given pair of acts

$$
f=\left[\begin{array}{cc}
L_{1} & \text { on } E_{1} \\
\vdots & \vdots \\
L_{n} & \text { on } E_{n}
\end{array}\right] \text { and } g=\left[\begin{array}{cc}
L_{1}^{\prime} & \text { on } E_{1}^{\prime} \\
\vdots & \vdots \\
L_{n^{\prime}}^{\prime} & \text { on } E_{n^{\prime}}^{\prime}
\end{array}\right]
$$

and apply the above methods, it follows from Axiom 3 (Mixture Monotonicity) that

$$
\begin{aligned}
f & \succsim g \text { if and only if } \\
{\left[\sum_{i=1}^{n-1}\left(U\left(L_{i}\right)-U\left(L_{i+1}\right)\right) \nu_{i}+U\left(L_{n}\right)\right] } & \geq\left[\sum_{j=1}^{n^{\prime}-1}\left(U\left(L_{j}^{\prime}\right)-U\left(L_{j+1}^{\prime}\right)\right) \nu_{j}^{\prime}+U\left(L_{n^{\prime}}^{\prime}\right)\right] .
\end{aligned}
$$

Hence, if we set, $\nu(\emptyset):=0, \nu(S):=1$ and $\nu\left(\cup_{j=1}^{i} E_{i}\right):=\nu_{i}$ then we have established that $\succsim$ can be represented by the Choquet expected utility functional

$$
\begin{aligned}
\operatorname{CEU}\left(\left[\begin{array}{cc}
L_{1} & \text { on } E_{1} \\
\vdots & \vdots \\
L_{n} & \text { on } E_{n}
\end{array}\right]\right) & =\sum_{i=1}^{n-1}\left(U\left(L_{i}\right)-U\left(L_{i+1}\right)\right) \nu\left(\cup_{j=1}^{i} E_{j}\right)+U\left(L_{n}\right) \\
& =U\left(L_{1}\right) \nu\left(E_{1}\right)+\sum_{i=2}^{n}\left(U\left(L_{i}\right)\right)\left(\nu\left(\cup_{j=1}^{i} E_{j}\right)-\nu\left(\cup_{j=1}^{i-1} E_{j}\right)\right)
\end{aligned}
$$

as required.

Finally we show (b) implies (a). Axioms 1-3 are immediate from the existence and form of the functional representation. Axiom 4 follows since for any pair of events $A, B \in \mathcal{E}$, and any two outcomes, $x$ and $y$ for which $U\left(\delta_{x}\right)>U\left(\delta_{y}\right)$,

$\mathrm{CEU}\left(\left[\delta_{x}\right.\right.$ on $B ; \delta_{y}$ on $\left.\left.\mathcal{S}-B\right]\right)-\mathrm{CEU}\left(\left[\delta_{x}\right.\right.$ on $A ; \delta_{y}$ on $\left.\left.\mathcal{S}-A\right]\right)=[\nu(B)-\nu(A)]\left[U\left(\delta_{x}\right)-U\left(\delta_{y}\right)\right] \geq 0$ 
Since $\nu$ is a capacity, $A \subset B$ implies $\nu(A) \leq \nu(B)$, which means $\operatorname{CEU}\left(\left[\delta_{x}\right.\right.$ on $B ; \delta_{y}$ on $\left.\left.\mathcal{S}-B\right]\right)$ $\geq \mathrm{CEU}\left(\left[\delta_{x}\right.\right.$ on $A ; \delta_{y}$ on $\left.\left.\mathcal{S}-A\right]\right)$, as required.

To see that Axiom 5 holds, notice that for the act $f=\left[L_{1}\right.$ on $E_{1} ; \ldots ; L_{n}$ on $\left.E_{n}\right]$, consider the act obtained by replacing $L_{j}$ on $E_{j}$ with $L_{j}^{\prime}$, where $L_{j}^{\prime} \sim L_{j}$, that is $U\left(L_{j}^{\prime}\right)=U\left(L_{j}\right)$. Observe that the act obtained by this replacement, is the act

$$
g=\left[L_{1} \text { on } E_{1} ; \ldots L_{j-1} \text { on } E_{j-1} ; L^{\prime} \text { on } E_{j} ; L_{j+1} \text { on } E_{j+1} ; \ldots ; L_{n} \text { on } E_{n}\right] .
$$

Hence

$$
\operatorname{CEU}(f)-\operatorname{CEU}(g)=\left(U\left(L_{j}\right)-U\left(L_{j}^{\prime}\right)\right)\left(\nu\left(\cup_{k=1}^{j} E_{k}\right)-\nu\left(\cup_{k=1}^{j-1} E_{k}\right)\right)=0
$$

as required. To see that Axiom 6 holds, consider any three pairwise comonotonic acts $f, g$ and $h$. Let $\left\{E_{1}, \ldots, E_{n}\right\}$ be the coarsest ordered partition with respect to which all three acts are measurable. That is, we can express $f=\left[L_{1}\right.$ on $E_{1} ; \ldots ; L_{n}$ on $\left.E_{n}\right], g=$ $\left[L_{1}^{\prime}\right.$ on $E_{1} ; \ldots ; L_{n}^{\prime}$ on $\left.E_{n}\right]$ and $h=\left[L_{1}^{\prime \prime}\right.$ on $E_{1} ; \ldots ; L_{n}^{\prime \prime}$ on $\left.E_{n}\right]$, where $L_{i} \succsim_{i} L_{i+1}, L_{i}^{\prime} \succsim_{i} L_{i+1}^{\prime}$ and $L_{i}^{\prime \prime} \succsim_{i} L_{i+1}^{\prime \prime}$, for all $i=1, \ldots, n-1$. Thus, for any $\alpha$ in $(0,1]$, we obtain

$$
\begin{aligned}
& \operatorname{CEU}(\alpha f+(1-\alpha) h)-\operatorname{CEU}(\alpha g+(1-\alpha) h) \\
= & {\left[U\left(\alpha L_{1}+(1-\alpha) L_{1}^{\prime \prime}\right)-U\left(\alpha L_{1}^{\prime}+(1-\alpha) L_{1}^{\prime \prime}\right)\right] \nu\left(E_{1}\right) } \\
& +\sum_{i=2}^{n}\left[U\left(\alpha L_{i}+(1-\alpha) L_{i}^{\prime \prime}\right)-U\left(\alpha L_{i}^{\prime}+(1-\alpha) L_{i}^{\prime \prime}\right)\right]\left(\nu\left(\cup_{j=1}^{i} E_{j}\right)-\nu\left(\cup_{j=1}^{i-1} E_{j}\right)\right) \\
= & \alpha U\left(L_{1}-L_{1}^{\prime}\right) \nu\left(E_{1}\right)+\sum_{i=2}^{n} \alpha U\left(L_{i}-L_{i}^{\prime}\right)\left(\nu\left(\cup_{j=1}^{i} E_{j}\right)-\nu\left(\cup_{j=1}^{i-1} E_{j}\right)\right) \\
= & \alpha(\operatorname{CEU}(f)-\operatorname{CEU}(g)) .
\end{aligned}
$$

And in particular this implies that if $f \sim g$ then $\alpha f+(1-\alpha) h \sim \alpha g+(1-\alpha) h$, as required.

\section{References}

Anscombe, F.J., Aumann, R.J: A Definition of Subjective Probability.

Annals of Mathematical Statistics 34, 199-205 (1963).

Fishburn, P.C.: Utility Theory for Decision Making.

Huntington, NY: Robert E. Kreiger Publishing Company 1979.

Gilboa, I.: Expected utility with purely subjective non-additive probabilities.

Journal of Mathematical Economics 16, 65-88 (1963).

Schmeidler, D.: Subjective probability and expected utility without additivity.

Econometrica 57, 571-587 (1989).

Savage, L.J.: The Foundations of Statistics.

New York: Dover Publications 1972 (first edition 1954). 